\title{
Microdiscectomia lombar tubular com o uso de seringas descartáveis de diâmetros progressivos: resultados cirúrgicos
} Tubular lumbar microdiscectomy using disposable syringes of progressive diameters: surgical results Microdiscectomía lumbar tubular con el uso de seringas desechables de diámetros progresivos: resultados quirúrgicos

\author{
Diogo Valli Anderle' \\ Andrei Fernandes Joaquim ${ }^{2}$ \\ Marcus Vinicius Giglio' \\ Enrico Ghizoni² \\ Luis Adriano Esteves ${ }^{2}$
}

\section{RESUMO}

Objetivo: descrever técnica minimamente invasiva para abordagem das hérnias discais com material de baixo custo. Métodos: estudo prospectivo da avaliação dos resultados cirúrgicos de dez pacientes submetidos à microdiscectomia lombar tubular com uso de seringas descartáveis de diâmetros progressivos. Resultados: utilizando os critérios de MacNab modificados, $60 \%$ dos pacientes apresentaram resultados considerados excelentes, $30 \%$ bons e $10 \%$ razoáveis. O tempo médio de cirurgia foi de 70 minutos e o de permanência hospitalar, de 14 horas. Conclusão: o uso de seringas descartáveis para microdiscectomia tubular foi seguro, de baixo custo e com resultados preliminares semelhantes aos da literatura para o tratamento minimamente invasivo da hérnia discal lombar.

\section{ABSTRACT}

Objective: to describe a minimally invasive technique to treat lumbar disc herniation with low-cost material. Methods: this is a prospective evaluation of ten patients submitted to a tubular lumbar microdiscectomy with disposable syringes for herniated disc. The modified MacNab criteria were used to evaluate the surgical results. Results: according to the modified MacNab criteria, surgical results were divided in: excellent in $60 \%$, good in $30 \%$ and reasonable in $10 \%$. The mean surgical time was 70 minutes and the mean hospital stay was 14 hours. Conclusion: the minimal invasive tubular microdiscectomy with disposable syringes is a feasible technique which obtains good results at a low cost, and the surgical results were similar to the recent literature about the treatment of herniated lumbar disc disease.
\end{abstract}

\section{RESUMEN}

Objetivo: describir una técnica mínimamente invasiva para el abordaje de las hernias discales con material de bajo costo y de fácil acceso. Métodos: estudio prospectivo de la evaluación de los resultados quirúrgicos de diez pacientes sometidos a una microdiscectomía lumbar utilizando jeringas desechables de diámetros progresivos. Resultados: utilizando los criterios de MacNab cambiados, un $60 \%$ de los pacientes tuvieron resultados considerados como excelentes, el $30 \%$ buenos y el $10 \%$ razonables. El tiempo medio de cirugía fue $70 \mathrm{mi}$ nutos y de permanencia en el hospital fue de 14 horas. Conclusión: los resultados del estudio indican que la técnica es viable, de bajo costo y con resultados similares a los reportados en la literatura para el tratamiento mínimamente invasivo de la hernia discal lumbar.

\footnotetext{
Santa Casa de Amparo - Amparo (SP), Brasil.

'Neurocirurgiões da Santa Casa de Amparo - Amparo (SP), Brasil e do Hospital Estadual de Francisco Morato "Professor Carlos da Silva Lacaz" - Francisco Morato (SP), Brasil.

${ }^{2}$ Neurocirurgiōes do Hospital Estadual de Francisco Morato "Professor Carlos da Silva Lacaz" - Francisco Morato (SP), Brasil.

Não há conflito de interesses.
} 


\author{
DESCRITORES: Discotomia/ \\ métodos; Vértebras lombares/ \\ cirurgia; Deslocamento do \\ disco intervertebral/cirurgia; \\ Procedimentos cirúrgicos \\ minimamente invasivos/ \\ métodos; Resultado de \\ tratamento
}

KEYWORDS: Diskectomy/

methods; Lumbar vertebrae/

surgery; Intervertebral

disk displacement/surgery;

Surgical procedures, minimally invasive/methods; Treatment outcome
DESCRIPTORES: Discectomía/

métodos; Vértebras lumbares/ cirugía; Desplazamiento del disco intervertebral/cirugía; Procedimientos quirúrgicos mínimamente invasivos/ métodos; Resultado del tratamiento

\section{INTRODUÇÃO}

O primeiro tratamento cirúrgico com sucesso para hérnia discal lombar ocorreu em 1934, $4^{1,2}$ Depois, houve modificações técnicas que permitiram o tratamento desta doença com melhores resultados e menor incidência de complicações. Um dos grandes avanços foi a utilização da discectomia com microscópio (microdiscectomia), introduzida em 1970 na Europa por Yasargil ${ }^{3}$ e Caspar $^{4}$ e nas Américas por Willians ${ }^{5}$ e Wilson e Kenn ${ }^{6,7}$, possibilitando minimizar a via de acesso com magnificacão e iluminacão adequadas do campo cirúrgico. Foley e Smith, em 1997, descreveram a abordagem microendoscópica com afastadores tubulares por via trasmuscular e a abordagem interlaminar ${ }^{8,9}$. Com o advento destas técnicas menos invasivas, vários autores publicaram seus resultados evidenciando incisões menores, redução do volume de sangramento intraoperatório e menos dor pós-operatória ${ }^{10-13}$. Modificações desta técnica microendoscópica foram descritas com o uso de afastadores metálicos progressivos e de diâmetros fixos ${ }^{14,15}$.

\section{OBJETIVO}

Descrever a técnica cirúrgica de microdiscectomia tubular com o uso de seringas de diâmetros progressivos para abordar, de forma pouco invasiva e com instrumentos cirúrgicos de baixo custo, a hérnia de disco lombar.

\section{MÉTODOS}

Estudo prospectivo de avaliação dos resultados cirúrgicos de pacientes com hérnia discal lombar submetidos à microdiscectomia tubular com seringas de diâmetros progressivos utilizadas como afastadores tubulares. Os critérios de inclusão foram: pacientes maiores de 18 anos que consentiram em realizar o procedimento cirúrgico, ciática refratária ao tratamento clínico adequado por seis semanas e secundária à hérnia discal lombar. Pacientes com hérnia discal operada previamente ou com estenose lombar (doença degenerativa lombar com múltiplas áreas de compressão) foram excluídos do trabalho.

Para avaliação dos resultados, o critério de MacNab (Figura 1) modificado foi utilizado.

Foi quantificado o tempo cirúrgico e o de permanência hospitalar. O termo de consentimento livre e esclarecido relacionado ao procedimento cirúrgico foi obtido junto aos dez pacientes no pré-operatório.

Descrição da técnica: foram empregadas seringas plásticas convencionais com os seguintes volumes: $1 \mathrm{~mL}, 3$
$\mathrm{mL}, 5 \mathrm{~mL}, 10 \mathrm{~mL}$ e $20 \mathrm{~mL}$. Essas seringas foram utilizadas como "afastadores tubulares", similarmente aos desenvolvidos por empresas de materiais cirúrgicos. Todos os pacientes foram submetidos à cirurgia com anestesia geral. O posicionamento cirúrgico foi genupeitoral. Após degermação, antisepsia (clorexedine degermante e alcoólico na degermação e antisepsia, respectivamente) e colocação dos campos esterilizados, foi realizada a marcação da pele no ponto de entrada à cerca de $2 \mathrm{~cm}$ da linha média, com o auxílio de fluoroscopia. Em perfil, identifica-se a união da lâmina com a faceta inferior da vértebra cranial, utilizando-se um pino de Steinmann. A marcação é centrada neste pino e, dependendo da quantidade de tecidos (muscular ou gorduroso) interpostos entre a pele e superfície óssea, uma incisão de 15 a $25 \mathrm{~mm}$ é suficiente. Seguese infiltração com anestésico local utilizando xilocaína $1 \%$ com vasoconstrictor na infiltração da pele e subcutâneo, e adrenalina, com abertura da pele e subcutâneo. Em seguida, a fáscia toracolombar é aberta, o pino de Steinmann é relocado e nova marcação fluoroscopia em perfil é realizada. Confirmado o ponto exato na superfície óssea, inicia-se a dilatação progressiva com as seringas de volume

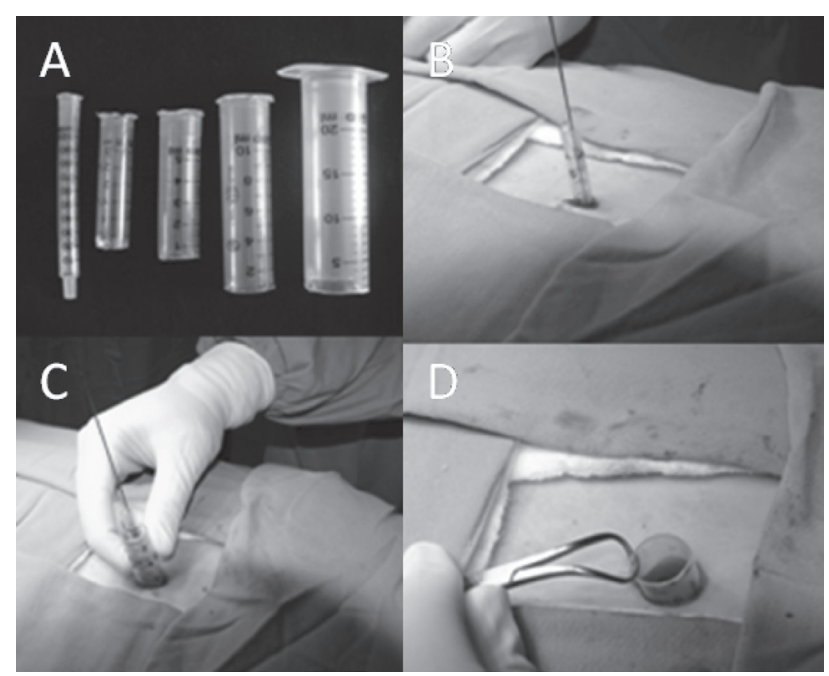

Figura 1

(A) Esquemas com seringas sucessivamente cortadas, funcionando similarmente aos dilatadores tubulares. (B) Início da dilatação, iniciada com pino de Steinmann após marcação radioscópica e seringa inicial de $1 \mathrm{~mL}$. (C) Progressão da dilatação e (D) aspecto final da área de trabalho com seringa de $10 \mathrm{~mL}$ sendo fixada pelo auxiliar com pinça Backaus. 
progressivamente crescente, usando o pino como guia, até que ela entre em contato com a superfície óssea. Retira-se o pino e realizam-se movimentos de báscula com a seringa com intuito de dissecar os tecidos moles.

Corta-se a extremidade distal deste tubo plástico e progride-se com as seringas previamente preparadas até os volumes de 10 ou de $20 \mathrm{~mL}$ (dependendo do volume de tecidos moles do paciente). Define-se a seringa que será usada como afastador final, deixando externalizado cerca de $1 \mathrm{~cm}$ na superfície da incisão.

Com o uso de pinças Backaus, o auxiliar orienta a inclinação do afastador nos sentidos medial, lateral, superior e inferior. Desta maneira, o cirurgião pode ter uma visão direta do espaço interlaminar, faceta inferior (cápsula articular), lâmina superior e inferior. Neste momento, pode ocorrer uma quantidade variável de tecido muscular aderido nas estruturas citadas acima, o qual é de fácil desbridamento com pinças de disco e coagulação bipolar. Caso exista dúvida em relação à localização do afastador, pode-se realizar novo controle radioscópico com dissector de Penfield n 3. Procede-se à ressecção óssea das lâminas com pinça Kerrinson ou drill de alta rotação. Em virtude do campo de visão limitado, a ressecção óssea é efetuada de acordo com a localização da hérnia discal ou de seu fragmento migrado. Abre-se o ligamento amarelo adjacente à superfície óssea ressecada, expondo-se a raiz comprometida e a hérnia discal. Não é exposto de forma rotineira o saco dural, evidenciando-se apenas a raiz no recesso lateral. Se necessário, coagula-se o plexo venoso sobre o espaço discal e a microdiscectomia com afastamento da raiz em questão é realizada pelo auxiliar. Após a realização da discectomia, sutura-se a fáscia toracolombar e o subcutâneo com vicryl 2.0, e a pele é aproximada com suturas intradérmicas com vycril 4.0 transparente. $O$ paciente tem alta hospitalar geralmente seis horas após o procedimento.

\section{RESULTADOS}

Dez pacientes foram submetidos ao procedimento. Os níveis operados foram L2-L3 ( $\mathrm{n}=1), \mathrm{L} 4-\mathrm{L} 5(\mathrm{n}=6)$ e L5-S1 $(n=3)$. Destes, apenas um paciente possuia hérnia foraminal (todos os demais tinham hérnias centro-laterais). Em relação à distribuição quanto ao sexo, seis eram do sexo masculino e quatro do sexo feminino.

O seguimento pós-operatório variou de dois a oito meses, com média de quatro meses. O tempo de cirurgia foi em média de 70 minutos, variando de 40 a 110 minutos, e o tempo de permanência hospitalar foi entre 6 e 20 horas (Tabela 1).

Os resultados cirúrgicos preliminares, conforme o critério de MacNab (Quadro1), em um seguimento médio relativamente curto de quatro meses, foi distribuído da seguinte maneira: seis dos pacientes obtiveram resultados considerados excelentes, três bons e um razoável (Tabela 2). Não ocorreram complicações intraoperatórias, como fístulas liquóricas, nem infecções no pós-operatório imediato ou tardio.

\section{QUADRO 1 - Critério de MacNab modificado para avaliar resultados clínicos dos dez pacientes submetidos à microdiscectomia tubular com seringas.}

\begin{tabular}{|c|c|}
\hline Excelente & $\begin{array}{l}\text { Livre de dor } \\
\text { Sem restrição de mobilidade } \\
\text { Apto a retornar às atividades normais e ao } \\
\text { trabalho }\end{array}$ \\
\hline Bom & $\begin{array}{l}\text { Dores não-radiculares ocasionais } \\
\text { Alívio dos sintomas prévios } \\
\text { Apto ao retorno ao trabalho adaptado }\end{array}$ \\
\hline Razoável & $\begin{array}{l}\text { Alguma melhora da capacidade funcional } \\
\text { Permanece incapacitado ou desempregado }\end{array}$ \\
\hline Ruim & $\begin{array}{l}\text { Permanência dos sintomas objetivos de } \\
\text { comprometimento radicular } \\
\text { Intervenções cirúrgicas adicionais necessárias } \\
\text { no nível acometido independentemente da } \\
\text { quantidade de reavaliações ou período de } \\
\text { seguimento }\end{array}$ \\
\hline
\end{tabular}

\section{TABELA 1 - Idade, sexo, tempo de cirurgia e de permanência hospitalar}

\begin{tabular}{lll}
\hline $\begin{array}{l}\text { Idade (anos) } \\
\text { Sexo }\end{array}$ & $\begin{array}{l}\text { De } 24 \text { a } 65 \text { anos } \\
6 \text { homens e } \\
4 \text { mulheres }\end{array}$ & Média: 32 anos \\
$\begin{array}{l}\text { Tempo de } \\
\text { permanência } \\
\text { hospitalar }\end{array}$ & $\begin{array}{l}\text { De } 6 \text { a } 20 \text { horas } \\
\begin{array}{l}\text { Tempo médio de } \\
\text { cirurgia }\end{array}\end{array}$ & $\begin{array}{c}\text { Média: } 14 \\
\text { horas }\end{array}$ \\
$\begin{array}{l}\text { Localização das } \\
\text { hérnias }\end{array}$ & 1 L2-3, 6 L4-5 e 3 L5-S1 10 minutos & $\begin{array}{l}\text { Média: } 70 \\
\text { minutos }\end{array}$ \\
\hline
\end{tabular}

\section{TABELA 2 - Resultados cirúrgicos dos dez pacientes segundo critérios de MacNab modificados}

\begin{tabular}{ll}
\hline Resultado & $\mathbf{n}(\%)$ \\
\hline Excelente & $6(60)$ \\
Bom & $3(30)$ \\
Razoável & $1(10)$ \\
\hline
\end{tabular}

Enfatiza-se que seis dos pacientes submetidos à cirurgia encontravam-se em litígios trabalhistas. $\mathrm{O}$ paciente com resultado razoável estava sob litígio trabalhista, embora não possa ser estabelecida relação causal.

\section{DISCUSSÃO}

O sistema de retrator tubular desenvolvido para uso com endoscópio foi modificado para utilização com microscópio, o que permite visualização tridimensional, pelo fato de haver familiaridade com a técnica pelo cirurgião de coluna com formação neurocirúrgica ${ }^{10,16}$.

Em estudo de Perez-Cruet et al., 150 pacientes foram submetidos à discectomia endoscópica tubular. 
Utilizando-se dos critérios de MacNab, 77\% dos pacientes tiveram excelentes resultados, $17 \%$ bons, 3\% razoáveis e apenas 3\% apresentaram resultados ruins. O tempo médio de hospitalização foi de sete horas. Embora a amostra seja pequena e com tempo de seguimento curto, nossa casuística teve resultados satisfatórios e similares aos dos autores $^{10}$.

Há consenso na literatura de que os bons resultados no tratamento cirurgico da hérnia discal lombar estão mais relacionados à seleção adequada do paciente e à correlação da clínica com os resultados dos exames de imagem do que propriamente ao tipo de técnica cirúrgica utilizada ${ }^{17}$. Dentre as cirurgias da coluna vertebral, a microdiscectomia está entre os procedimentos de resultados mais satisfatórios. As abordagens tradicionais, com dissecção muscular subperiosteal, causam maior sangramento, dor e tempo de internação em relação a técnicas menos invasivas ${ }^{12}$. As abordagens minimamente invasivas, por proporcionarem acesso através da musculatura paravertebral, a qual é afastada excentricamente, tem o benefício de lesar menos os tecidos paravertebrais ${ }^{18}$. Estudos evidenciaram menor liberação de marcadores séricos de lesão tecidual, como a proteína C-reativa e citocinas nas cirurgias ditas minimamente invasivas ${ }^{19}$. No entanto, os resultados clínicos a longo prazo entre as técnicas aberta e tubulares não evidenciam diferenças estatisticamente significativas ${ }^{13}$. Outro estudo controlado, randomizado e duplo-cego não evidenciou melhor resultado da técnica tubular minimamente invasiva em relação à aberta após um ano de seguimento, obtendo, pelo contrário, uma taxa maior de bons resultados (79 versus $69 \%$ ) com a técnica de microdiscectomia convencional ${ }^{20}$. Acrescenta-se que dispositivos tecnólogicos para realização de técnicas minimamente invasivas estão disponíveis, porém têm alto custo, o que inviabiliza seu uso rotineiro e limita o número de estudos clínicos ${ }^{8,16}$.

\section{CONCLUSÃO}

O crescente uso de técnicas menos invasivas é uma tendência natural da medicina moderna. Nossa técnica é uma alternativa viável para o alto custo desses procedimentos, com maior acessibilidade. Embora o tempo de seguimento tenha sido curto, nossos resultados encorajam novos estudos clínicos mais bem desenhados com o método para estabelecer sua real eficácia.

\section{REFERÊNCIAS}

1. Mixter WJ, Barr JS. Rupture of intervertebral disc with involvement of the spinal canal. N Engl J Med. 1934;211:210-4.

2. Parish K, Tomasino A, Knopman J, Boockvar J, Härtl R. Operative results and learning curve: microscopeassisted tubular microsurgery for 1- and 2-level(s) discectomies and Laminectomies. Neurosurg Focus. 2008;25(2):E14.

3. Yasargil MG. Microsurgical operation of herniated lumbar disc. Adv Neurosurg. 1977;4:81-2.

4. Caspar W. A new surgical procedure for lumbar disc herniation causing less tissue damage through a microsurgical approach. Adv Neurosurg. 1977;4: 74-80.

5. Williams RW. Microlumbar discectomy: a conservative surgical approach to the virgin herniated lumbar disc. Spine. 1978;3(2): 175-82.

6. Wilson DH, Kenning J. Microsurgical lumbar discectomy: preliminary report of 83 consecutive cases. Neurosurgery. 1979;4(2):137-40.
7. Jaikumar S, Kim DH, Kam AC. Histoyy of minimally invasive spine surgery. Neurosurgery. 2002;51(Suppl 2):1-14.

8. Foley KT, Smith MM. Microendoscopic discectomy. Tech Neurosurg. 1997;3:301-7.

9. Perez-Cruet MJ, Smith M, Foley K. Microendoscopic lumbar discectomy. In: Perez-Cruet MJ, Fessler RG, editors. Outpatient spinal surgery. St. Louis: Quality Medical Publishing; 2002. p. 171-83.

10.Perez-Cruet MJ, Foley KT, Isaacs RE, Rice-Wyllie L, Wellington R, Smith MM, et al. Microendoscopic lumbar discectomy: technical note. Neurosurgery. 2002;51

(5 Suppl):S129-36.

11. Brayda-Bruno M, Cinnella P. Posterior endoscopic discectomy (and other procedures). Eur Spine. 2000;9 Suppl 1:S24-9.

12.German JW, Adamo MA, Hoppenot RG, Blossom JH, Nagle HA. Perioperative results following lumbar discectomy: comparison of minimally invasive discectomy and standard microdiscectomy. Neurosurg Focus. 2008;25(2):E20.
13.Righesso O, Falavigna A, Avanzi O. Comparison of open discectomy with microendoscopic discectomy in lumbar disc herniations: results of a randomized controlled trial. Neurosurgery. 2007;61(3):545-9.

14.Armin SS, Holly LT, Khoo LT. Minimally invasive decompression for lumbar stenosis and disc herniation. Neurosurg Focus. 2008;25(2):E11.

15.Ryang YM, Oertel MF, Mayfrank L, Gilsbach JM, Rohde V. Standard open microdiscectomy versus minimal access trocar microdiscectomy: results of a prospective randomized study. Neurosurgery. 2007;62(1):174-82.

16. Thongtrangan I, Le H, Park J, Kim DH. Minimally invasive spinal surgery: a historical perspective. Neurosurg Focus. 2004;16(1):E13.

17.Choi PS, Junior RB. Estudo anatômico da zona triangular de segurança aplicada aos procedimentos percutâneos póstero-laterais lombares. Coluna. 2003;2(1):20-6. 
18.Stevens KJ, Spenciner DB, Griffiths KL, Kim KD, Zwienenberg-Lee M, Alamin T, et al. Comparison of minimally invasive and conventional open posterolateral lumbar fusion using magnetic resonance imaging and retraction pressure studies. J Spinal Disord Tech. 2006;19(2):77-86.
19.Huang TJ, Wen-Wei Hsu R, Li YY, Cheng CC. Less systemic cytokine response in patients following microendoscopic versus open lumbar discectomy. J Orthop Res. 2005;23(2):406-11

20.Arts MP, Brant R, Akker E, Koes BW, Bartels RHMA, Peul WC, et al. Tubular diskectomy versus conventional microdiskectomy for sciatica: A randomized controlled trial. JAMA. 2009;302(2):149-58.

\section{Correspondência:}

Diogo Valli Anderle

Avenida Bernardino de Campos, 173, $2^{\circ}$ andar

CEP: 13900-400 - Amparo (SP), Brasil

E-mail:vallianderle@gmail.com 